\title{
Metabolism of sphingolipids in experimental obesity and insulin resistance
}

\author{
Saatov T.S., Zainutdinov B.R., Ibragimova E.A.,Irgasheva S.U., Tashmanova A.B.,Mustafakulov M.A. \\ Institute of Bioorganic Chemistry, Uzbekistan Academy of Sciences, Tashkent Pediatric Medical Institute
}

\section{Objectives:}

\section{Methods:}

\section{Results:}

Experimental model of obesity and insulin resistance was used to study activity of sphingomyelinase as well as concentrations of sphingosine and ceramide. Increase in activity of sphingomyelinase was found in the liver and skeletal muscles of the animals. Significant alterations in the content of sphingomyelin and its metabolites were observed in obese rats; these alterations were found to be oppositely directed. In the liver of rats with experimental obesity sphingomyelin was found to decrease by $25 \%$; while in skeletal muscles sphingomyelin decreased more than by $31 \%$. As to ceramide and sphingosine, their concentrations in the liver of obese rats were found to increase significantly (by $15 \%$ and $23 \%$, respectively), as compared with the controls. In skeletal muscle of rats with experimental obesity concentrations of ceramide and sphingosine were observed to increase by $19 \%$ and $68 \%$, respectively.

Increase in the activity of sphingomyelinase and accumulation of ceramide and sphingosine, metabolites of sphingomyelin, was observed in the liver and skeletal muscles of rats with experimental obesity and insulin resistance.

Ceramide overproduction plays a key role in the onset and progression of insulin resistance.
Sphingolipids or "bioefficient lipids" constitute the structural base for all types of biological membranes, and are numerous in human and animal tissues. Metabolites of sphingolipids act as significant biological effectors, modulators and mediators in a number of biochemical processes; they are known to be pathogens in various human pathologies

Prolonged high-calories diet was used to produce obesity and insulin resistance model in rats. Total lipids ceramide were measured by methods of Lauter and Trems; activity of acid and neutral sphing was measured by the modified method of Hostetler and Yazaki. 